\title{
Prótese para substituição total de disco intervertebral: desenvolvimento de modelo computacional e análise por elementos finitos
}

\author{
Prosthesis for total intervertebral disc replacement: computacional model \\ development and finite element analysis
}

Prótese de reemplazo total del disco intervertebral: modelo de desarrollo computacional y análisis de elementos finitos

\author{
Tiago Nunes Campello' \\ Isaac Newton Lima da Silva ${ }^{2}$ \\ Marcelo Simoni Simões ${ }^{3}$
}

\section{RESUMO}

Introdução: a idéia de um disco intervertebral artificial não é nova. $\mathrm{O}$ campo de estudos sobre próteses para artroplastia de coluna desenvolve-se ao passo que novas tecnologias na área de materiais e engenharia médica são desenvolvidas ou introduzidas para o surgimento de novos projetos. Objetivo: estabelecer metodologia de desenvolvimento de produto em um projeto de prótese para substituição total de disco intervertebral pela utilização de ferramentas computacionais de engenharia. Métodos: a metodologia de desenvolvimento de prótese para substituição total de disco iniciou-se com a definição do seu modelo virtual, seguida pela a análise mecânica virtual por elementos finitos. Resultados: a prótese de disco foi concebida com três componentes, sendo eles o flange superior, o flange inferior e o núcleo. Aplicando o critério de von Mises para solução da análise virtual, verificou-se que o núcleo da prótese é o componente mais solicitado durante compressão axial e compressão/

\section{ABSTRACT}

Introduction: the idea of an artificial intervertebral disc is not new. The studies of spinal arthroplasty prostheses are in developing, while new materials and technologies are developed to suply medical engineering projects. Objective: to establish a methodology for product development on a project of prosthesis for total disc replacement, trough the use of computacional engineering tools. Methods: the methodology of prosthesis development began with the definition of their virtual model, followed by the virtual mechanical analysis by finite element. Results: the prosthesis is designed with three components: the top flange, bottom flange and the core. Applying the von Mises criterion for the virtual analysis solution, it was found that the core of the prosthesis is the most stressed component during axial compression and compression/shear. Conclusion: this study demonstrated that the development of a total disc replacement prosthesis is viable

\section{RESUMEN}

Introducción: la idea de un disco intervertebral artificial no es nueva. Los estudios de la columna vertebral artroplastia prótesis están en desarrollo, mientras las nuevas tecnologías en el ámbito de la ingeniería y los materiales médicos son desarr llados o introducidos a la aparición de nuevos proyectos. Objetivo: establecer una metodología para el desarrollo de producto en un proyecto de prótesis para reemplazo total del disco intervertebral, a través del uso de herramientas de ingeniería computacional. Métodos: la metodología de desarrollo de prótesis para reemplazo total del disco se inició con la definición de su modelo virtual, seguido por el virtual mecánica de análisis de elementos finitos. Resultados: la prótesis discal, fue diseñado con tres componentes, que son la parte superior de brida, la brida inferior y núcleo. Aplicando el criterio de von Mises solución virtual para el análisis, se constató que el núcleo de la prótesis es la más solicitada durante

\footnotetext{
Trabalho originalmente apresentado como dissertação de mestrado em Engenharia e Tecnologia de Materiais, na Faculdade de Engenharia da Pontifícia Universidade Católica do Rio Grande do Sul - PUCRS - Porto Alegre (RS), Brasil.

Não há conflitos de interesse.

'Engenheiro Mecânico e Mestre em Engenharia e Tecnologia de Materiais pela Pontifícia Universidade Católica do Rio Grande do Sul - PUCRS - Porto Alegre (RS), Brasil.

2Doutor, Professor da Faculdade de Engenharia da Pontifícia Universidade Católica do Rio Grande do Sul - PUCRS - Porto Alegre (RS), Brasil.

${ }^{3}$ Mestre em Engenharia e Tecnologia de Materiais pela Pontifícia Universidade Católica do Rio Grande do Sul - PUCRS - Porto Alegre (RS), Brasil. 
cisalhamento. Conclusão: este estudo demonstra a viabilidade do desenvolvimento de um projeto para fabricação de prótese para substituição total de disco intervertebral, por meio de metodologia computacional já consagrada em projetos mecânicos de engenharia, principalmente, nos ramos automotivo e aeronáutico.

DESCRITORES: Coluna lombar; Artroplastia; Desenho de prótese; Disco intervertebral; Projeto assistido por computador through computational methods already known in mechanical engineering projects, mainly in automotive and aeronautics.

KEYWORDS: Lumbar spine; Arthroplasty; Prosthesis design; Intervertebral disc; Computer aided design la compresión axial y la compresión y cortante. Conclusión: por último, este estudio demuestra la viabilidad de elaborar un proyecto para la construcción de una prótesis para reemplazo total del disco intervertebral, a través de métodos computacionales ya consagrados en los proyectos de ingeniería mecánica, principalmente en automoción y sector aeroespacial.

DESCRIPTORES: Columna lumbar; Artroplastia; Diseño de prótesis; Disco Intervertebral; Diseño asistido por computador

\section{INTRODUÇÃO}

A idéia de um disco intervertebral artificial não é nova. $O$ campo de estudos sobre próteses para artroplastia de coluna cresce, ao passo que novas tecnologias na área de materiais e engenharia médica são desenvolvidas ou adotadas para o surgimento de novos projetos ${ }^{1}$. Principalmente a partir de 1980, diversas técnicas de instrumentação de coluna vertebral foram desenvolvidas e aplicadas em pacientes por todo o mundo. Na década subsequente, houve um refinamento dos métodos cirúrgicos e um crescimento focado, principalmente, em técnicas minimamente invasivas. Apesar destes avanços, ainda existe uma disparidade entre os resultados dos métodos desenvolvidos e os resultados que seriam ideais para os pacientes ${ }^{1}$.

Dentro deste contexto, a idéia de artroplastia de coluna vertebral, com o desenvolvimento de próteses que preservem o movimento ou restaurem a funcionalidade do segmento motor problemático constitui-se no próximo e expressivo passo na área de cirurgia de coluna ${ }^{1}$.

O objetivo deste trabalho foi estabelecer uma metodologia de desenvolvimento de produto em um projeto de prótese para substituição total de disco intervertebral. Para isto, foram utilizadas ferramentas computacionais, que permitiram a modelagem virtual de uma prótese, e também a análise do seu comportamento mecânico quando submetida a esforços de compressão axial e compressão/cisalhamento estáticos, por meio de programa de Computer Aided Engineering (CAE) Ansys Workbench ${ }^{\mathrm{TM}}$, utilizando o Método dos Elementos Finitos para solução dos cálculos e aplicando nas simulações mecânicas virtuais as condições de contorno propostas pelas normas internacionais vigentes. Por meio desta metodologia de desenvolvimento computacional de produto, pretendemos viabilizar o projeto para fabricação da prótese de coluna lombar.

\section{MÉTODOS}

O desenvolvimento da prótese total de disco foi feito totalmente em ambiente computacional com o software Computer Aided Design (CAD) Autodesk Inventor ${ }^{\mathrm{TM}}$. A avaliação inicial do projeto geométrico e dimensional da prótese, ainda em fase de desenvolvimento no CAD, foi realizada pela análise crítica por médicos cirurgiões de coluna, que possuem conhecimento das possíveis complicações de natureza mecânica relacionadas à implantação de próteses de disco. Os materiais que compõem o projeto do modelo em CAD estão em conformidade com as normas da American Society for Testing and Materials (ASTM), sendo a liga de Cobalto-Cromo-Molibdênio em conformidade com a norma ASTM F75 Standard Specification for Cobalt-28 Chromium-6 Molybdenum Alloy Castings and Casting Alloy for Surgical Implants, e o Polietileno de Altíssimo Peso Molecular, em conformidade com a normaASTM F648StandardSpecification for Ultra-High-Molecular Weight Polyethylene Powder and Fabricated Form for Surgical Implants. Estes materiais foram selecionados por possuírem propriedades biomecânicas adequadas e porjá serem utilizados na fabricação de próteses ortopédicas.

Para a realização das análises mecânicas virtuais foi utilizado o software de CAE. Com esta ferramenta de engenharia, já consagrada na indústria mecânica de manufatura, é feita uma discretização do modelo virtual construído anteriormente em CAD em partes muito pequenas, chamadas de elementos finitos. Essa separação de peças complexas em vários elementos simplificados, possibilita obter soluções de cálculos complexos de engenharia, resolvendo-se um conjunto de equações algébricas para obtenção dos resultados desejados em função das solicitações, da geometria e dos dados mecânicos e físicos dos materiais. Esta discretização em elementos finitos é feita por meio da ge- 
ração de uma malha computacional, que tem fundamental importância nos cálculos computacionais ${ }^{2-3}$.

Para a realização das simulações computacionais utilizouse, como referência, a norma ASTM F2346 Standard Test Methods for Static and Dynamic Characterization of Spinal Artificial Discs. Primeiramente, foram realizadas análises mecânicas de compressão axial estática com carga de 2510N. Este valor força foi adotado como parâmetro de resistência mínima durante as análises, porque que durante atividades fisicas cotidianas, as forças geradas na coluna lombossacra são de aproximadamente 4,5 vezes maiores do que as encontradas em um indivíduo em repouso, que são descritas com o valor aproximado de $445 \mathrm{~N}$ em L5-S1 ${ }^{4}$. Posteriormente, realizaramse análises mecânicas de compressão/cisalhamento, onde a força foi aplicada com um vetor angulado $45^{\circ}$ na face superior externa do dispositivo de teste, com valor de $500 \mathrm{~N}$ em direção póstero-anterior. $\mathrm{O}$ valor de $500 \mathrm{~N}$ é, aproximadamente, o da carga gerada quando o disco intervertebral sofre uma compressão axial de $2510 \mathrm{~N}$ na região lombossacra, já que o espaço intervertebral nesta região da coluna não é paralelo à linha horizontal ${ }^{5-7}$.

\section{RESULTADOS}

A prótese de disco foi concebida com três componentes, sendo eles: o flange superior, o flange inferior e o núcleo (Figura 1). $\mathrm{O}$ flange superior e o flange inferior possuem geometrias semelhantes, diferenciando-se apenas nas superfícies de contato com o núcleo. As superfícies de contato do flange superior e inferior com os corpos vertebrais possuem cinco saliências, que foram inseridas para que haja uma correta ancoragem do dispositivo no osso cortical dos corpos vertebrais, evitando-se, desta maneira, um possível afrouxamento durante esforços mecânicos de cisalhamento e cíclicos. Para fabricação deste produto, também deverá ser considerada a deposição de material bioativo nas superfícies externas dos flanges, também para proporcionar maior aderência da prótese junto ao tecido ósseo ${ }^{8}$.

A saliência do centro dos flanges foi desenvolvida de forma a auxiliar na centralização da prótese no trans-operatório. Todas as âncoras foram modeladas com formato piramidal, pois, desta forma, estas contêm pontas agudas e arestas de corte que facilitam as suas inserções nos corpos vertebrais.

Nas suas superfícies de contato com o núcleo de polietileno, os flanges possuem características geométricas bem distintas e que determinam, juntamente com as características do núcleo, o mecanismo de articulação da prótese (Figura 2).

O núcleo é o ponto de articulação da prótese. Quando implantado, fica inserido entre o flange superior e inferior e tem formato cilíndrico, contendo em sua porção inferior uma superfície plana e na sua parte superior, uma convexidade esférica (Figura 3).

O contato esférico entre o flange superior e o núcleo permite movimentos de inclinação lateral e flexão/extensão da prótese, que ocorrem de forma não constrita, exceto pelos efeitos de interação com as estruturas esqueléticas e musculares após a implantação. A amplitude de movimento angular

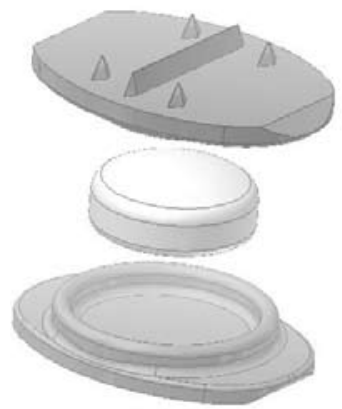

(A)

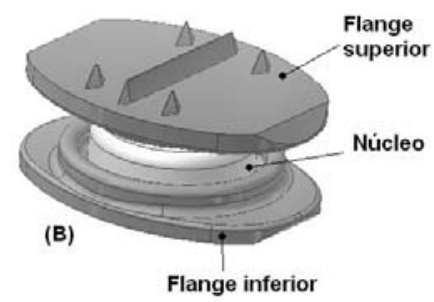

Figura 1

Modelo da prótese. Em (A) pode-se ver a prótese em vista explodida e em (B) a prótese montada e descrição de seus componentes

(A)

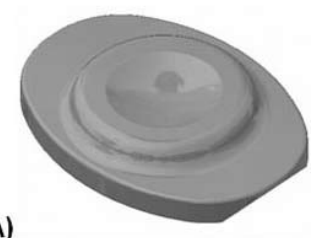

Figura 2

Superfícies internas dos flanges. Pode-se observar a concavidade existente no flange superior (A). Flange inferior com guarnição circular com interior plano (B)

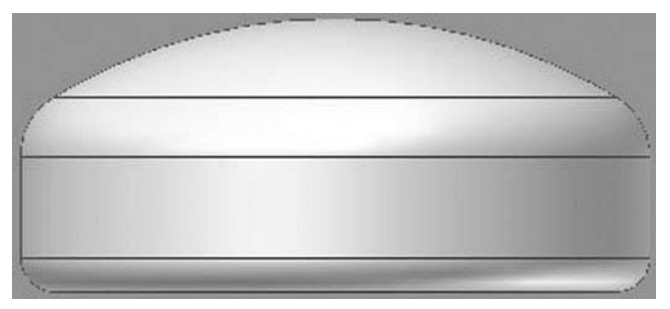

Figura 3 Núcleo da prótese
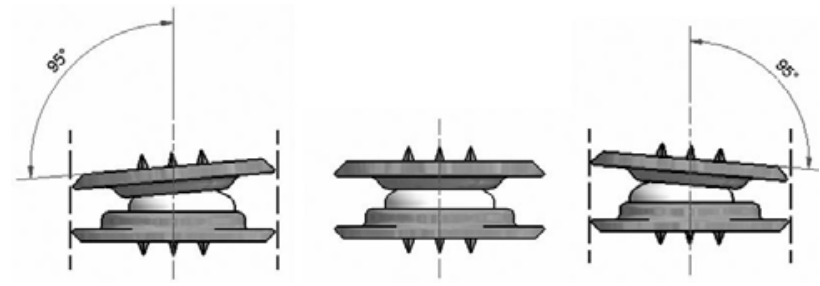

Figura 4

Prótese em configuração de movimento em inclinação lateral (vista frontal). Amplitude total do movimento angular igual a $10^{\circ}$
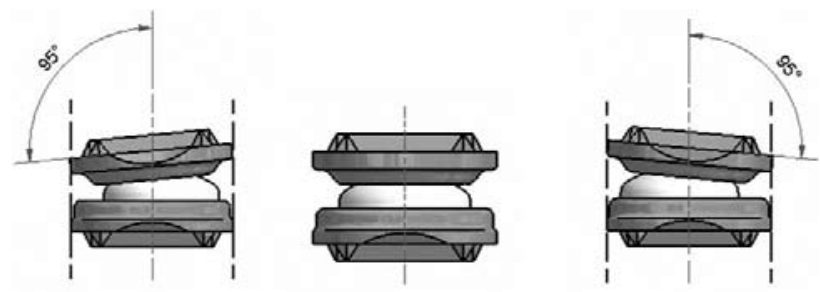

Figura 5

Prótese em configuração de movimento em flexão-extensão (vista lateral). Amplitude total do movimento angular igual a $10^{\circ}$ 
possível sem desalinhamento dos flanges, ou seja, sem translação dos corpos vertebrais, é de $10^{\circ}$ (Figuras 4 e 5). O núcleo da prótese tem liberdade para realizar movimento de translação devido a diferença entre o seu diâmetro e o diâmetro interno da guarnição do flange inferior. Isto é previsto para que haja a compensação natural do movimento de translação existente em um segmento motor de coluna, também mantendo a propriedade de translação do centro de rotação do segmento6. Esta possibilidade de translação também é fundamental para permitir o alinhamento vertical dos flanges durante a flexão/extensão e inclinação lateral, reduzindo a sobrecarga sobre as juntas facetárias ou mesmo evitando o desgaste desigual das facetas, como ocorre nos implantes com centro de rotação fixo.

Dentro do ambiente do software de CAE geraram-se as condições de teste definidas pela norma ASTM F 2346 para as análises mecânicas de compressão axial estática, e os dados das propriedades físicas dos materiais selecionados para a prótese foram aplicados (Figura 6). Para solução da análise virtual foi aplicado o critério de von Mises, que considera o seguinte: enquanto os valores de tensão equivalente encontrados nos testes virtuais não ultrapassarem a tensão de escoamento do material, este não perderia a sua funcionalidade no caso de implantação in vivo, pois as deformações sofridas não seriam significativas e permanentes, mas sim deformações menores que $2 \%$, dentro do limite elástico do material (deformações elásticas).

Verificou-se que o núcleo de polietileno da prótese é o componente mais solicitado durante compressão axial, sendo encontrado um valor máximo de tensão equivalente de $12,93 \mathrm{MPa}$, localizado na borda da calota convexa do núcleo da prótese, para uma força aplicada de $2510 \mathrm{~N}$. Esta tensão equivalente encontrada é menor do que a tensão de escoamento do material definido para este componente da prótese, o polietileno de altíssimo peso molecular, que é de 22,3MPa. Após diversos cálculos realizados, inclusive fazendo refinamentos da malha nas regiões de concentração de tensões, foi determinada a força máxima suportada pela prótese discal pelo critério de von Mises. Esta força tem o valor aproximado de 10100N (Figura 7).

A região de concentração de tensões se dá no núcleo, e é formada pelo contato direto das calotas esféricas do núcleo e do flange superior. Porém, esta situação foi oportunamente prevista em projeto, sendo criado um raio de suavização na borda da calota esférica do flange superior, para evitar um contato em forma de aresta entre o flange e o núcleo durante a compressão da prótese (Figura 8). Por fim, fez-se análise mecânica em esforços de compressão/cisalhamento. Nestas análises, a força foi aplicada com um vetor angulado $45^{\circ}$ na face superior externa do dispositivo de teste, com valor de $500 \mathrm{~N}$ em direção póstero-anterior. Novamente o critério de von Mises foi utilizado para solução dos cálculos virtuais. Verificou-se que, aplicando-se uma força proposta por Smith, Fernie5 para a região lombossacra, gera-se uma tensão equivalente de von Mises igual 19,11MPa, inferior ao valor da tensão de escoamento do polietileno de altíssimo peso molecular. Prosseguiu-se novamente com as simulações até que se encontrasse o valor de força máxima. Após diversos cálculos realizados, foi determinada a força máxima aproximada em $735 \mathrm{~N}$, para uma tensão equivalente de 22,04MPa.
Novamente pôde-se observar que o núcleo da prótese suportou cargas com valores além dos limites estabelecidos na literatura médica como gerados na coluna lombar em atividades cotidianas. (Figura 9)

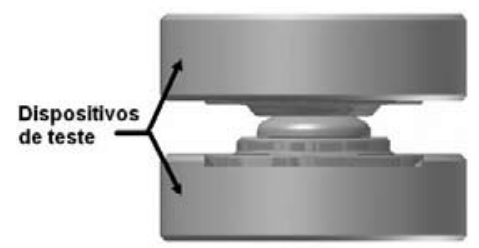

(A)

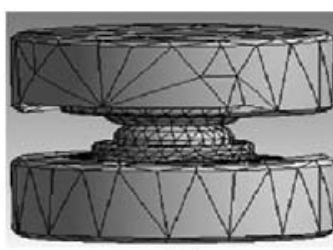

(B)

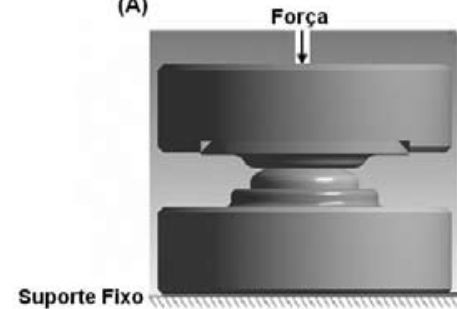

(C)

Figura 6

(A) Modelo em CAE, no qual podem ser observados os dispositivos de teste em conformidade com a norma ASTM F-2346. (B) Malha gerada para simulação. (C) Condições de contorno para análise computacional de esforços de compressão axial

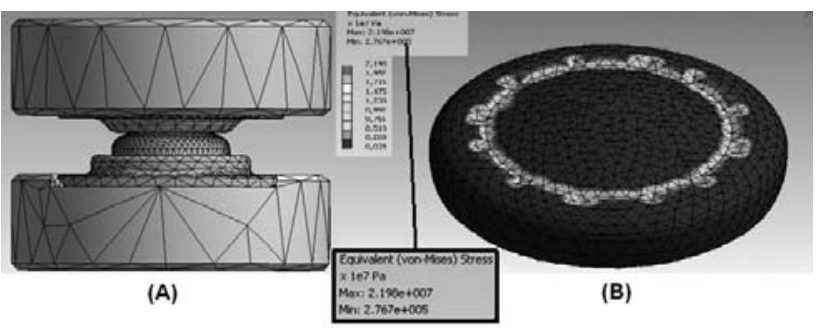

Figura 7

(A)Refinamento da malha feito no núcleo da prótese. (B) Zona de concentrações de tensões com valor máximo em $21,98 \mathrm{MPa}$ para uma força de $10100 \mathrm{~N}$

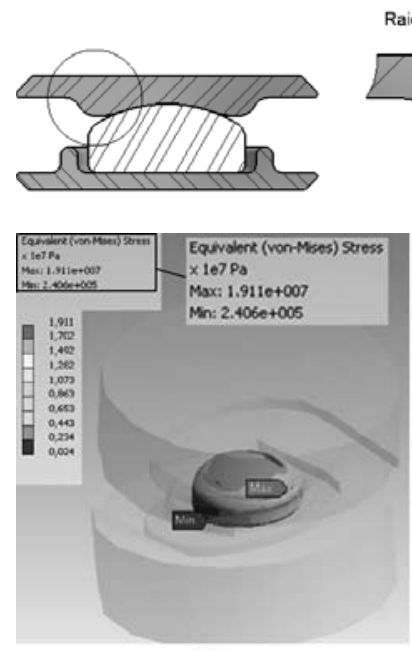

(A)

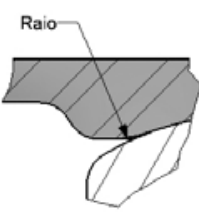

Figura 8 Raio de suavização inserido no flange superior

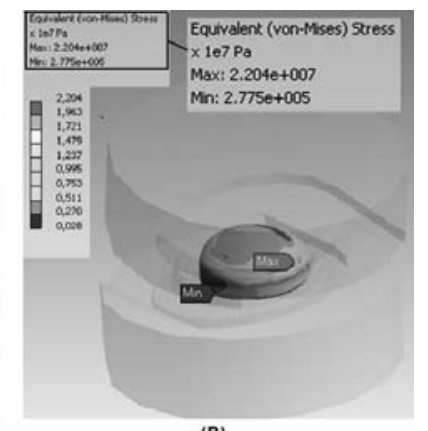

(B)
Figura 9

Resultados obtidos para as análises de esforços em compressão/ cisalhamento. A) Resultado encontrado para uma força de 500 N; B) Resultado para uma força aplicada de $735 \mathrm{~N}$ 


\section{DISCUSSÃO}

Realizado totalmente em ambiente virtual, este estudo apresenta a fundamental importância da utilização de ferramentas computacionais de engenharia em desenvolvimento de produto. A utilização interativa dos softwares de CAD e CAE auxilia o projeto, basicamente sob o seguinte ponto de vista:

- Economiza tempo e custos, pelo fato de ser possível analisar as características geométricas e dimensionais do produto antes de ocorrer investimentos em ferramentas e processos de fabricação;

- Possibilita a análise e diagnóstico de falhas mecânicas no produto sob condições simuladas de utilização, permitindo a melhoria do seu projeto ainda no modelo em CAD, e antes de serem realizados testes experimentais em laboratório;

- E, por fim, as análises mecânicas computacionais permitem uma visualização de concentrações de tensões geradas no produto quando submetidos a cargas, que nem sempre é possível de ser feita em tempo real em laboratório.

Analisando-se as simulações realizadas percebeu-se a coerência dos resultados encontrados, pois se esperava, ainda em fase de desenho, que a maior concentração de tensões se daria no núcleo da prótese, o que se verificou claramente nas simulações. Ainda durante os cálculos computacionais, também se pôde verificar a boa repetiçãobilidade encontrada ao se processar diversas configurações de malhas e refinamentos. De certa forma, a morfologia das cargas mecânicas estabelecidas nas condições de ensaio da norma simplificou os cálculos. É importante citar que, como os cálculos computacionais foram realizados com as condições de ensaio da norma, os seus resultados podem ser diretamente comparados com trabalhos experimentais que venham a ser realizados em laboratório.

Todavia, sabe-se que os esforços biomecânicos a serem considerados em projetos de implantes ortopédicos podem ser definidos como estáticos e cíclicos. Os esforços biomecânicos de origem cíclica causam fadiga nos mate- riais implantados, que é um dos modos de falha mais crítica observada em próteses para substituição total de disco intervertebral. Entretanto, os resultados previamente apresentados demonstram análises mecânicas computacionais apenas para esforços estáticos, e sem considerar fatores estruturais dos materiais que sejam iniciadores de falha por fadiga. Por isso, mesmo que os resultados deste estudo tenham sido satisfatórios, análises complementares devem ser realizadas para que o projeto da prótese esteja de acordo com critérios para utilização in vivo.

\section{CONCLUSÃO}

Desde a sua concepção, o desenvolvimento do modelo da prótese de disco foi realizado em ambiente computacional, facilitando a visualização e avaliação do modelo da prótese em cada etapa do desenvolvimento de seus componentes, inclusive proporcionando o melhor entendimento da equipe médica durante a fase inicial do modelo, o que foi fator decisivo para o bom andamento deste projeto realizado com uma equipe multidisciplinar.

A utilização de modelos CAD também é um pré-requisito para a geração do programa de usinagem por Computer Aided Manufacturing (CAM), necessário para a usinagem de peças complexas, como é o caso dos flanges da prótese.

As características geométricas, dimensionais e mecânicas da prótese foram avaliadas durante as simulações computacionais, demonstrando-se que esta possui resistência mecânica acima do necessário para a aplicação pretendida, quando considerados esforços estáticos. Também foi possível concluir que a escolha dos materiais considerados para a composição da prótese foi adequada e coerente com a finalidade de uso do produto.

E, por fim, este estudo demonstra a viabilidade do desenvolvimento de um projeto para fabricação de uma prótese para substituição total de disco intervertebral, por meio de metodologia computacional já consagrada em projetos mecânicos de engenharia, principalmente++ nos ramos automotivo e aeronáutico.

\section{REFERÊNCIAS}

1. Guyer RD, Zigler JE. Spinal arthroplasty - A new era in spine care. Sant Louis: Equality Medical Publishing; 2005.

2. Assan AE. Método dos elementos finitos: primeiros passos. Campinas: Editora da UNICAMP; 2003.

3. Adams V, Askenazi A. Building better products with finite element analysis. Santa Fe: Onword Press; 1998.

4. Wilke HJ, Neef P, Caimi M, Hoogland T, Claes LE. New in vivo measurements of pressures in the intervertebral disc in daily life. Spine. 1999 Apr 15;24(8):755-62.
5. Smith TJ, Fernie GR. Functional biomechanics of the spine. Spine. 1991;16(10):1197-203.

6. Panjabi MM, White III AA. Clinical biomechanics of the spine. 2nd ed. Philadelphia: Lippincott Williams \& Wilkins; 1990.

7. Tencer AF. Surgery of spinal trauma. Philadelphia: Lippincott Williams \& Wilkins; 2000.

8. Fujisawa A, Noda I, Nishio Y, Okimatsu H.. The development of new titanium arc-sprayed artificial joints. Mater Sci Eng Biomim Mater Sens Syst. 1995; 2(3): 151-7.

\author{
Correspondência \\ Tiago Nunes Campello \\ Rua Delfina Fusquini Sirianni, 60 \\ Jardim Vila Nova - Porto Alegre - RS \\ CEP: $91750-280$ \\ Tel.: + $55513266.3739 / 9693.9799$ \\ E-mail:campello150@yahoo.com.br
}

